\title{
Measurement-Based Modeling of Time-Variant Fading Statistics in Indoor Peer-to-Peer Scenarios
}

\author{
Evgenii Vinogradov, Wout Joseph, Claude Oestges
}

\begin{abstract}
Investigation of small-scale fading in indoor peerto-peer networks based on radio channel measurements shows that Rayleigh or double-Rayleigh fading with or without lineof-sight (LOS) component can occur, depending on the mobility and on the scattering properties of the environment. While in double-mobile scenarios, the predominant fading mechanism is a combination of Rayleigh and double-Rayleigh fading, Rician fading and fading events caused by combination of a constant term with a Double Rayleigh component are also occasionally observed. In single-mobile scenarios, temporal fading is Rician or Rayleigh distributed. Additionally, measurements reveal that fading statistics evolve significantly over time, even for smallscale motions of the nodes. We model the transitions between the fading states by means of a hidden Markov model, parameterized from our measurements. Eventually, we propose a complete simulation model that generates channel realizations by combining measurement-based first-order statistics with geometry-based second-order statistics for both types of node mobility.
\end{abstract}

Index Terms-Indoor propagation, Fading channels, Multipath channels, Propagation measurements, Time-varying channels.

\section{INTRODUCTION}

The demand for flexible mobile multimedia services continues to grow. Traditional cellular solutions are coming under increasing pressure from wireless peer-to-peer (P2P) systems. Many methods were introduced, where nodes accord assistance to other nodes in traffic maintenance. Popular schemes are relaying techniques [1], distributed space-time coding [2], or distributed beam-forming [3]. Underlying radio channels influence the performance of all of these schemes. Most works on cooperative communications assume stationary Rayleigh fading between the nodes. While this is often true in outdoor cellular scenarios, it is not the case in indoor peer-to-peer channels. Here, we observe a strong dependence on the node mobility and on the scattering scenario [4]. For this reason, the non-stationary peer-to-peer radio channel must be measured and modeled, as time-variant fading statistics may be exploited by incentive mechanisms based on repeated games that force nodes to help disadvantaged ones in view of a future exchange of roles, along the lines of [5].

Naturally, indoor radio propagation channels have been investigated in the past. In [6], various properties of indoor peer-

E. Vinogradov and C. Oestges are with the Electrical Engineering Department, Institute for Information and Communication Technologies, Electronics and Applied Mathematics (ICTEAM), Université catholique de Louvain, 1348 Louvain-la-Neuve, Belgium (e-mail: Evgenii.Vinogradov@UCLouvain.be)

W. Joseph is with Ghent University/iMinds, Gaston Crommenlaan 8 box 201, B-9050 Ghent, Belgium

This research is funded by the Interuniversity Attraction Poles Programme $7 / 23$ BESTCOM initiated by the Belgian Science Policy Office. It is also carried out in the framework of the FP7 Network of Excellence NEWCOM\# and COST Action IC 1004. to-peer channels have been analyzed, but for static nodes only: fading was modeled for single-antenna links by a generalized gamma distribution. Single mobile links between the nodes located in the same room (or adjacent rooms) under LOS and non-LOS conditions were investigated in [7]: fading was found to be Weibull distributed. In [4], peer-to-peer channels were empirically modeled in a typical cubicle-style office environment. The same environment was also used in [8] to investigate the multi-user separation in dual-link multipleinput and multiple-output (MIMO) indoor scenarios.

In this paper, we investigate narrowband indoor peer-to-peer channels based on a wideband experimental campaign at 3.8 $\mathrm{GHz}$ in a typical office environment consisting in different rooms aligned along corridor and separated by different types of walls (in contrast to [4], where a single large room with partitions was considered). Key contributions are as follows.

- We analyze and model the variations of small-scale fading statistics based on measurements and using the so-called second-order scattering fading (SOSF) distribution [9], [4] which reflects any combination of Rician, Rayleigh, and double-Rayleigh fading [10]. In addition, secondorder statistics are derived using a double-ring model [11]. The combination of empirical first-order statistics and geometry-based channel dynamics enables to implement a time-series generator of channel realizations.

- To model the sudden changes of fading statistics, we introduce a three-step approach: (i) for reflecting the sudden changes of the statistics, we define and parameterize a hidden Markov model [12], (ii) the parameters of the SOSF distribution are modeled according to a Beta or an Extreme Value distribution, fitting the measurements, (iii) fading realizations are generated [13].

This paper is organized as follows. In Section II, we introduce the measurements on which our work is based. Section III introduces the approach to represent non-stationary fading statistics and details the empirical model parameters.The proposed equivalent geometry-based model for second-order scattering fading (SOSF) is presented in Section IV. Section V describes the path-loss and shadowing models extracted from the measurements and provides an implementation summary and simulation results of the model, which is validated against measurements.

\section{Measurements}

\section{A. Environment}

This paper is based on channel measurements carried out at the Université Catholique de Louvain (UCL), Louvain-la- 
TABLE I

Measurement Parameters (After Post-Processing)

\begin{tabular}{cc}
\hline Parameter & Value \\
\hline Center frequency & $3.8 \mathrm{GHz}$ \\
Transmit power & $23 \mathrm{dBm}$ \\
Measurement bandwidth (null-to-null) & $50 \mathrm{MHz}$ \\
Bandwidth (post-processed) & $30 \mathrm{MHz}$ \\
Recorded frequency tones $F$ & 121 \\
Recorded time samples $N_{s}$ & 300 \\
Measurement duration & $113 \mathrm{~s}$ \\
Code length & 2047 \\
\hline
\end{tabular}

Neuve, Belgium, in Fall 2009. The investigated environment was located on the first floor of an office building, and consisted of typical offices along a corridor separated by brick or plaster-board walls, as illustrated in Fig. 1. Circles indicate mobile nodes (moving in random directions with the walking speed $v \approx 1 \mathrm{~m} / \mathrm{s}$ ), squares indicate static nodes; receivers are additionally marked by a cross. Two different types mobility scales were investigated: over a small area (within a square of $1 \mathrm{~m}^{2}$ ) and over a larger area (throughout the whole room they were in). Depending on the node mobility, peer-to-peer channels were measured for four sub-scenarios :

- single-mobile links (where only one node is moving),

- double-mobile links (where both nodes are moving),

- links between static nodes(in the absence of moving people in corridors)

- links between static nodes(where a few people moved along the corridors).

For links between static nodes (in the absence and presence of moving people in corridors), channel statistics were investigated in [14]. Hence, this paper only considers links between mobile nodes.

\section{B. Equipment}

The measurements were carried out with UCL/ULB Elektrobit PROPSound ${ }^{\mathrm{TM}} \mathrm{CS}$ at a carrier frequency of $3.8 \mathrm{GHz}$, using the switched-array principle. The sounder was calibrated

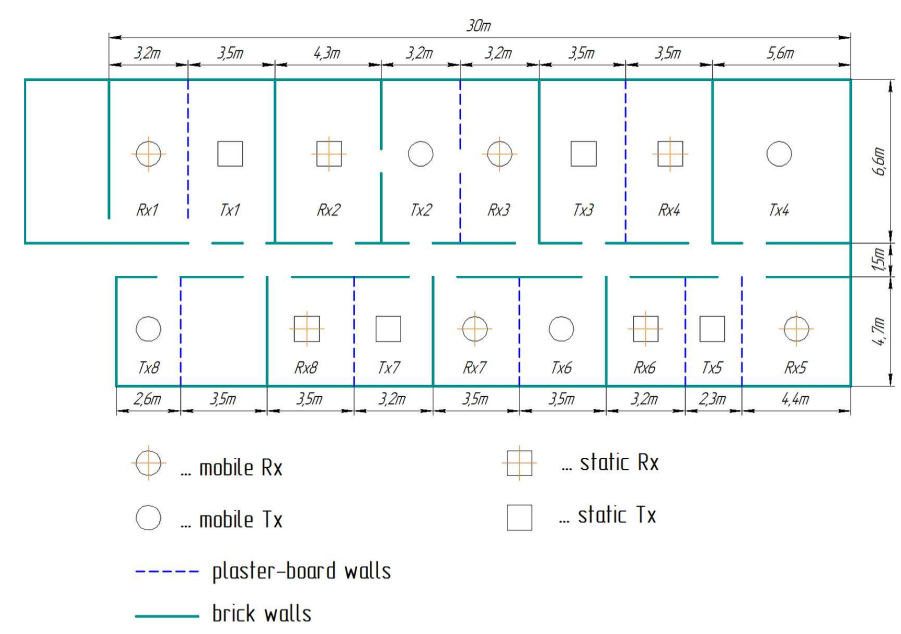

Fig. 1. Floor-plan of peer-to-peer measurements
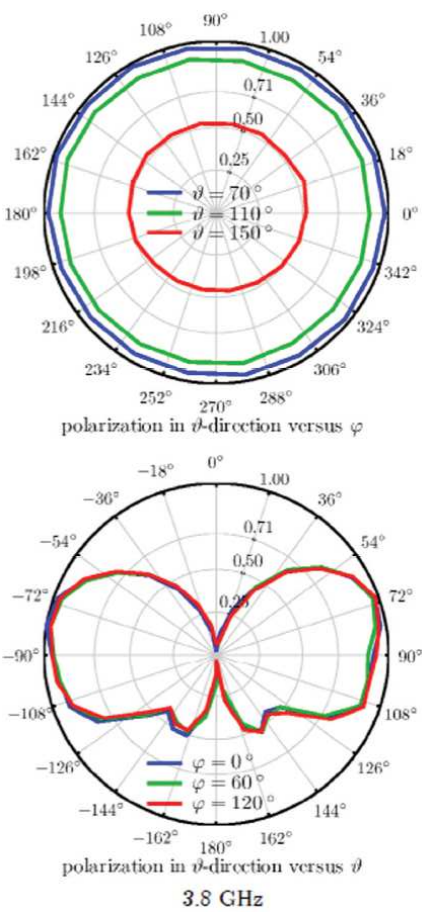

Fig. 2. Radiation pattern of the node antenna

twice (before and after measuring channels). The distributed nodes were connected to the 8-port switches of the transmitter and receiver of the channel sounder using long low-loss RF cables, which had equal length. The RF cables had excellent RF stability, even when they were slightly bent or moved during the measurements. At the nodes, custom-made dipole antennas with a gain of $1.75 \mathrm{~dB}$ and an almost omnidirectional radiation pattern (see Fig.2) were used. The channel sounder used long pseudo-noise (PN) sequences to estimate the impulse response of the radio channels between all combination of $\mathrm{Tx}$ and $\mathrm{Rx}$ nodes. The measurement parameters are summarized in Table I. The recorded channel transfer function is denoted by $H[t, f, j]$, where $t$ denotes the time index, $f$ denotes the frequency index, and $j$ denotes the link index (one link joining one transmit node to a receive node).

\section{Concepts of Data Analysis}

The channel results from the addition (when expressed in logarithmic scale, i.e. in decibels) of path-loss $\Lambda$, shadowing $S(t)$ and small-scale fading $G(t)$. The path loss is constant over time for a given link, and only depends on the distance between the nodes, whereas shadowing and fading are timevariant. Let us define the average received power as

$$
\bar{P}[j]=\frac{1}{N_{s} F} \sum_{t=1}^{N_{s}} \sum_{f=1}^{F}|H[t, f, j]|^{2}
$$

where $t$ and $f$ denote the time sample and the frequency tones respectively.

It turns out that the maximum stationarity time of fast fading equals $0.8 \mathrm{~s}$. Accordingly, the length of the sliding time window $T_{a v}$ used to define the time-variant average power as 
the received power averaged over all tones was chosen to be 20 time samples (corresponding to $\frac{113 \mathrm{~s}}{300} \cdot 20=7.5 \mathrm{~s}$ ) and the size of sliding shift of the time window equals 2 , so that the time-variant average power can be written as

$$
P[t, j]=\frac{1}{T_{a v} F} \sum_{t^{\prime}=t-T_{a v} / 2}^{t+T_{a v} / 2} \sum_{f=1}^{F}\left|H\left[t^{\prime}, f, j\right]\right|^{2}
$$

Consequently, we obtain $T=\frac{N_{s}-T_{a v}+2}{2}=141$ realizations of $P[t, j]$ which allow us to evaluate the time-variant average power each $\frac{113 \mathrm{~s}}{141}=0.8 \mathrm{~s}$.

Based on these two definitions, we may evaluate

- the path-loss exponent $\eta$ by fitting the log-log variation of $\bar{P}[j]$ over the average distance $\bar{d}_{j}$ between the nodes of link $j$,

$$
\left.\left.\bar{P}[j]\right|_{d B} \rightarrow \bar{P}_{0}\right|_{d B}-\eta \cdot 10 \log _{10}\left(\frac{\bar{d}_{j}}{d_{0}}\right),
$$

where $\bar{P}_{0}$ denotes the reference power at the reference distance $d_{0}=1 \mathrm{~m} \mathrm{[15],} \mathrm{[16],}$

- the shadowing (in decibels)

$$
S[t, j]=\left.\bar{P}_{0}\right|_{d B}-\eta \cdot 10 \log _{10}\left(\frac{\bar{d}_{j}}{d_{0}}\right)-\left.P[t, j]\right|_{d B},
$$

- the small-scale fading (in natural scale)

$$
G[t, f, j]=\frac{H[t, f, j]}{\sqrt{P[t, j]}},
$$

the fading amplitude being then simply defined as $g=$ $|G|$.

To extract first-order statistics of time-frequency fading, $T$ blocks of $T_{a v} \times F$ of realizations can be used.

For a given link $j$, shadowing can be expressed as the sum of a mean value and a time-variant component, so that $S[t, j]=\bar{S}[j]+\tilde{S}[t, j]$. Mean shadowing $\bar{S}[j]$ is related to time-invariant site-specific obstructions of the link including the influence of different effects such as refraction, diffraction, reflection, and absorption in environment (furniture, static people etc.). Hence, $\bar{S}[j]$ is a random variable depending on the environment. Dynamic shadowing $\tilde{S}[t, j]$ is caused by the mobility of scatterers and so-called body shadowing (due to the changing orientation of the person holding the device). Additionally, when nodes are moving over a large scale, shadowing caused by the mobility of the stations themselves cannot be considered as static unlike the case of small scale motions of the nodes. Therefore, we expect the standard deviation $\sigma_{\tilde{s}}$ to increase in the case of large scale motion. Dynamic shadowing can also be expressed as

$$
\tilde{S}[t, j]=-\left[\left.P[t, j]\right|_{d B}-\bar{S}[j]\right] .
$$

Note that we can normalize fading so that $\mathbb{E}\left\{g^{2}\right\}=1$, the expectation being conducted over time or frequency.

\section{EXPERIMENTAL CHARACTERIZATION OF Non-STATIONARY TIME-FrequenCy FADING STATISTICS}

\section{A. The SOSF Distribution}

It is shown in [4] that, for peer-to-peer environments, smallscale fading over time and/or frequency can be described by a

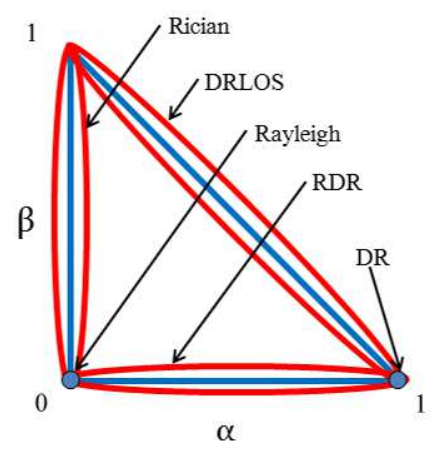

Fig. 3. Triangle of the parameters of SOSF distribution

single distribution including a weighted combination of a lineof-sight (LOS) component, a Rayleigh fading component and a Double Rayleigh fading component. Hence, any realization of the time-frequency channel can be expressed as

$$
G(t, f)=\omega_{0} e^{j \theta}+\omega_{1} G_{1}(t, f)+\omega_{2} G_{2}(t, f) G_{3}(t, f)
$$

where $G_{1}, G_{2}, G_{3}$ are i.i.d. complex normal random variables with zero mean and unit variance, and $\theta$ is a constant phase shift angle in $[0,2 \pi]$. The probability density function of $g=$ $|G|$ is given, as shown in [17] and [4], by the so-called second order scattering fading (SOSF) distribution

$$
\rho_{S O S F}(g)=\int_{0}^{\infty} \omega e^{-\omega_{1}^{2} \omega^{2} / 4} \frac{4 J_{0}(g \omega) J_{0}\left(\omega_{0} \omega\right)}{4+\omega_{2}^{2} \omega^{2}} d \omega
$$

where $J_{0}$ is the Bessel function of the first kind and zeroth order.

It can be useful to derive simpler forms for distribution functions in special cases of the SOSF distribution. Probability density functions of Double Rayleigh (DR) and RayleighDouble Rayleigh (RDR) distributions can be found in [17] and [9], respectively. The distribution for a combination of Double Rayleigh and LOS (DRLOS) can be derived by using the same concept as used in [17] and the standard integral [18, $\S 6.541]$ as

$$
\rho_{D R L O S}(g)=\frac{4 g}{\omega_{2}^{2}} I_{0}\left(\frac{2 a_{1}}{\omega_{2}}\right) K_{0}\left(\frac{2 a_{2}}{\omega_{2}}\right)
$$

where $I_{0}$ and $K_{0}$ are the modified Bessel functions of the first kind and zeroth order and the modified Bessel functions of the second kind and zeroth order, respectively, parameters $a_{1}$ and $a_{2}$ are given by

$$
\begin{aligned}
& a_{1}= \begin{cases}g & {\left[g<\omega_{0}\right]} \\
\omega_{0} & {\left[g \geq \omega_{0}\right]}\end{cases} \\
& a_{2}= \begin{cases}\omega_{0} & {\left[g<\omega_{0}\right]} \\
g & {\left[g \geq \omega_{0}\right]}\end{cases}
\end{aligned}
$$

Since $\mathbb{E}\left\{g^{2}\right\}=1$, we have that $\omega_{0}^{2}+\omega_{1}^{2}+\omega_{2}^{2}=1$ and the distribution can be specified by only two parameters [19]

$$
\begin{aligned}
& \alpha=\frac{\omega_{2}^{2}}{\omega_{0}^{2}+\omega_{1}^{2}+\omega_{2}^{2}} \\
& \beta=\frac{\omega_{0}^{2}}{\omega_{0}^{2}+\omega_{1}^{2}+\omega_{2}^{2}}
\end{aligned}
$$




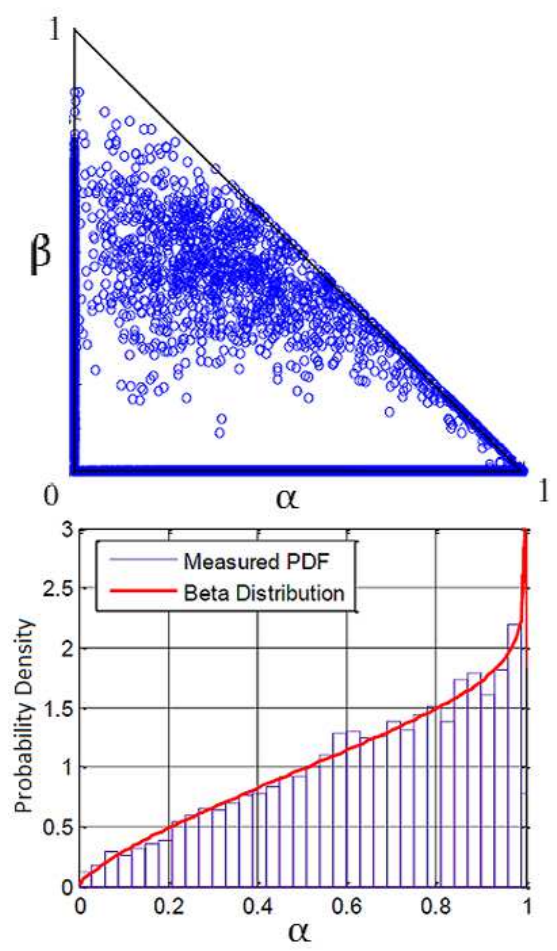

Fig. 4. Measured parameters of SOSF realizations for Double mobile (top); probability density function of the parameter $\alpha$ (bottom)

TABLE II

PROBABILITIES OF THE SUBSETS

\begin{tabular}{l|l|l}
\hline Subset & $\begin{array}{l}\text { Single } \\
\text { mobile }\end{array}$ & $\begin{array}{l}\text { Double } \\
\text { mobile }\end{array}$ \\
\hline Rician & 0.12 & 0.05 \\
Rayleigh & 0.03 & 0.02 \\
RDR & 0.78 & 0.86 \\
Double Rayleigh & 0.05 & 0.06 \\
DRLOS & 0.02 & 0.01 \\
\hline
\end{tabular}

where $(\alpha, \beta)$ are constrained to the triangle $\alpha \geq 0, \beta \geq 0$, and $\alpha+\beta \leq 1$ shown in Fig. 3. In specific cases of the triangle, the SOSF distribution reduces to Rician $(\alpha=0, \beta>0$, with $K$ Factor $\left.K=\frac{\beta}{1-\beta}\right)$, Rayleigh $(\alpha=0, \beta=0)$, Double Rayleigh $(\alpha=1, \beta=0)$, DRLOS $(\alpha+\beta=1, \alpha<1, \beta<1)$ and Rayleigh-Double-Rayleigh distribution $(0<\alpha<1, \beta=0)$. Based on previous studies [20], [4], we can expect that these distributions describe predominant fading mechanisms for our environment.

\section{B. SOSF Parameters Extracted From Measurements}

The time-varying parameters $\alpha$ and $\beta$ presented in (10) and (11) can be estimated from experimental time-frequency fading realizations by iteratively fitting the SOSF probability density function assuming a moment-based estimate as starting point [19].

Looking at the values of $\alpha(t)$ and $\beta(t)$ in Fig. 4 (top), we observe that the five groups presented in Section III-A describe the major part of measured fast fading realizations. For reasons of simplicity, we neglect points in the middle zone
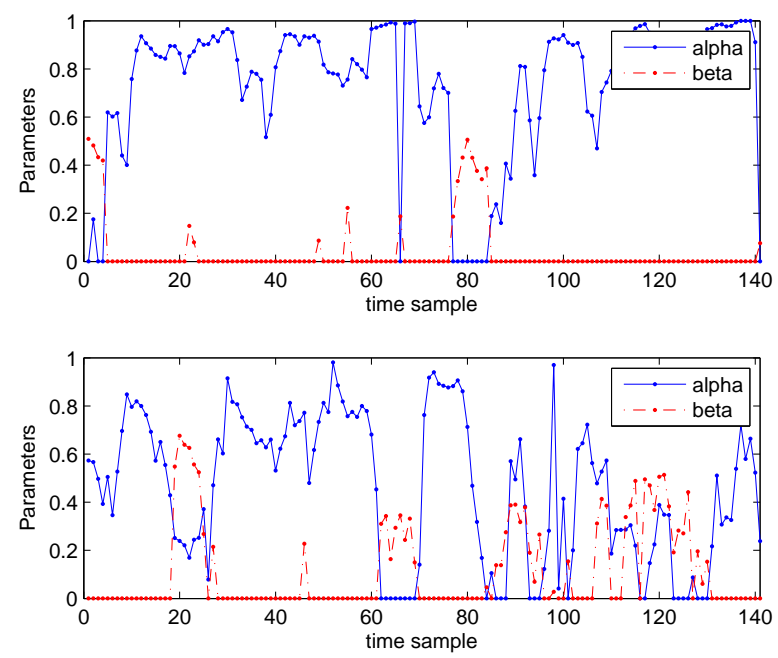

Fig. 5. Typical variation of small-scale time-frequency fading parameters over time for double-mobile Tx2-Rx3 (top) and single-mobile Tx1-Rx7 (bottom).

TABLE III

EVALUATED PARAMETERS OF THE TIME-FREQUENCY SOSF DISTRIBUTION FOR DIFFERENT MOBILITY SCENARIOS

\begin{tabular}{l|c|c}
\hline \multirow{2}{*}{ Subset } & Single Mobile & Double mobile \\
\cline { 2 - 3 } & Distribution & Distribution \\
\hline Rician & $K \sim p_{\text {ev }}(-1.15,3.6), \alpha=0$ & $K \sim p_{e v}(-1.83,3.68), \alpha=0$ \\
RDR & $\alpha \sim p_{\beta}(1.8,0.85), \beta=0$ & $\alpha \sim p_{\beta}(2.08,0.79), \beta=0$ \\
DRLOS & $\Delta \sim p_{\beta}(1,1.9)$ & $\Delta \sim p_{\beta}(1.32,2.73)$ \\
\hline
\end{tabular}

(where $0<\alpha<1,0<\beta<1$ and $\alpha+\beta \neq 1$ ), which occur only with a very low probability $(\sim 1 \%)$.

Fig. 5 (top) shows time-variant changes of the parameters $\alpha(t)$ and $\beta(t)$ for a typical double-mobile channel. We see that predominantly Rayleigh-double-Rayleigh fading $(\beta=0,0<$ $\alpha<1$ ) occurs. Only scarcely, we observe Rician fading, pure double-Rayleigh fading or DRLOS fading. Looking at the single-mobile channel in Fig. 5 (bottom), we observe that Rayleigh-double-Rayleigh fading remains the main fading mechanism. However, Rayleigh fading $(\alpha=0, \beta=0)$ and Rician fading $(\alpha=0,0<\beta<1)$ occur with a higher probability than in the case of the double-mobile channel.

The distribution of the parameter $\alpha$ for the Rayleigh-double Rayleigh subset (see Fig. 4) is found to be well approximated by the Beta distribution

$$
p_{\beta}\left(x \mid p_{1}, p_{2}\right)=\frac{\Gamma\left(p_{1}+p_{2}\right)}{\Gamma\left(p_{1}\right) \Gamma\left(p_{2}\right)} x^{p_{1}-1}(1-x)^{p_{2}-1}
$$

where $\Gamma(\cdot)$ denotes the Gamma function. For the DRLOS distribution, we define $\Delta=\sqrt{(1-\alpha)^{2}+\beta^{2}}$, denoting the set of points on the line $\alpha+\beta=1$. Parameter $\Delta$ is also found to be Beta-distributed. Finally, the distribution of the K-Factor in Rician fading, $K=\frac{\beta}{1-\beta}$, can be modeled by Extremal Value distribution,

$$
p_{e v}(x \mid \mu, \sigma)=\sigma^{-1} e^{\left(\frac{x-\mu}{\sigma}\right)} e^{\left(-e^{\left(\frac{x-\mu}{\sigma}\right)}\right)}
$$

when the K-factor expressed in decibels.

Eventually, probabilities of the subsets and a probability distribution of the SOSF parameters within the subset can be 
TABLE IV

VALUES OF THE PARAMETER $\tau$ FOR THE AUTOREGRESSIVE MODEL

\begin{tabular}{c|c|c|c}
\hline & \multicolumn{3}{|c}{ Parameter } \\
\hline Subscenario & $\tau_{K}$ & $\tau_{\alpha}$ & $\tau_{\Delta}$ \\
\hline Double Mobile & $1.1 \mathrm{~s}$ & $3.1 \mathrm{~s}$ & $1.3 \mathrm{~s}$ \\
\hline Single Mobile & $1.4 \mathrm{~s}$ & $2.8 \mathrm{~s}$ & $1 \mathrm{~s}$ \\
\hline
\end{tabular}

estimated(see Tables II and III). Note, that a high probability of occurrence for Rayleigh - double Rayleigh fading is observed $(78-86 \%)$.

The temporal autocorrelations of the parameters $\alpha(t), \beta(t)$ and $\Delta(t)$ can be modeled by a decreasing exponential function. Hence, an autoregressive process can be used to generate autocorrelated values of the parameters of a SOSF channel over time samples $(t=[1, \cdots, T])$ :

$$
\begin{gathered}
\alpha(t)=e^{-1 / \tau_{\alpha}} \alpha(t-1)+\sqrt{1-e^{-2 / \tau_{\alpha}}} x_{\alpha}(t) \\
\Delta(t)=e^{-1 / \tau_{\Delta}} \Delta(t-1)+\sqrt{1-e^{-2 / \tau_{\Delta}}} x_{\Delta}(t) \\
K(t)=e^{-1 / \tau_{K}} K(t-1)+\sqrt{1-e^{-2 / \tau_{K}}} x_{K}(t)
\end{gathered}
$$

where $x_{\alpha}, x_{\Delta}, x_{K}$ are time series of length $T$, whose values are drawn independently from the distributions described by Table III. The parameters $\tau$ are extracted from measurements and given in Table IV.

Using modeled values of the parameters $\alpha$ and $\beta$, the weights in (7) can be calculated as

$$
\begin{aligned}
\omega_{0}=\sqrt{\beta} & =\sqrt{\frac{K}{1+K}} \\
\omega_{1}=\sqrt{1-\alpha-\beta} & =\sqrt{1-\alpha-\frac{K}{1+K}} \\
\omega_{2} & =\sqrt{\alpha}
\end{aligned}
$$

\section{Hidden Markov Model}

Transitions between different fading states can be modeled by a hidden Markov model (HMM) [12]. Usually, the system being modeled by a HMM is assumed to be a Markov process with unobserved (hidden) states. Since the SOSF distribution reduces to the five distributions of small-scale fading highlighted above, we model the transitions between the subsets using a five-state HMM: (i) Rican fading, (ii) Rayleigh- double-Rayleigh fading (iii) pure double-Rayleigh fading, (iv) pure Rayleigh fading and (v) DRLOS fading.

Using measured data, the state transition probabilities have been estimated for the four sub-scenarios (see Table V)

\section{Double-Ring Models For Peer-to-Peer Radio Channels}

Our ultimate goal is to create a radio channel simulator

\begin{tabular}{|c|c|c|c|c|c|}
\hline \multicolumn{6}{|c|}{ Single mobile } \\
\hline Subset & Rician & RDR & DRLOS & $\begin{array}{l}\text { Double } \\
\text { Rayleigh }\end{array}$ & Rayleigh \\
\hline Rician & 0.77 & 0.19 & 0.01 & 0 & 0.03 \\
\hline RDR & 0.03 & 0.93 & 0.01 & 0.02 & 0.01 \\
\hline DRLOS & 0.05 & 0.44 & 0.50 & 0.01 & 0 \\
\hline Double Rayleigh & 0 & 0.24 & 0.01 & 0.75 & 0 \\
\hline Rayleigh & 0.13 & 0.40 & 0 & 0.01 & 0.46 \\
\hline \multicolumn{6}{|c|}{ Double mobile } \\
\hline Subset & Rician & RDR & DRLOS & $\begin{array}{l}\text { Double } \\
\text { Rayleigh }\end{array}$ & $\overline{\text { Rayleigh }}$ \\
\hline Rician & 0.68 & 0.26 & 0.01 & 0.01 & 0.04 \\
\hline RDR & 0.02 & 0.95 & 0.01 & 0.01 & 0.01 \\
\hline DRLOS & 0.03 & 0.60 & 0.36 & 0.01 & 0 \\
\hline Double Rayleigh & 0 & 0.29 & 0.01 & 0.70 & 0 \\
\hline Rayleigh & 0.10 & 0.54 & 0 & 0 & 0.36 \\
\hline
\end{tabular}
taking into account both first- and second-order statistics of small-scale fading. However, the measurement setup does not permit to extract appropriate second-order statistics. For this
TABLE V

TRANSITION MATRICES

reason, we need to propose an equivalent representation that is compatible with measured first-order statistics but further enables to model second-order statistics. Geometrically, each room along the corridor can be represented by a local ring of scatterers around a given node. As the nodes were located in different rooms, the corresponding geometry of any given link can indeed be represented by two different rings of scatterers.

\section{A. Generalized Reference Double-Ring Model for SOSF chan- nels}

The double-ring geometry [11] used to fit a reference model to the SOSF formalism is illustrated in Fig. 6. Transmitter (Tx) and receiver $(\mathrm{Rx})$ are denoted by $0_{T}$ and $0_{R}$, respectively; $R_{T}$ and $R_{R}$ are radii of rings which are formed by scatterers $S_{T}^{m}(m=1,2, \ldots M)$ and $S_{R}^{n}(n=1,2, \ldots N)$ located around the transmitter and the receiver. The symbols $\gamma_{T}^{m}$ and $\gamma_{R}^{n}$ denote the angle of departure (AOD) of the $m$-th transmitting wave and the angle of arrival (AOA) of the $n$-th receiving wave, respectively. The following assumptions are made: (i) the nodes move with speed $v_{T}$ and $v_{R}$ in the direction determined by the angle of motion $\Theta_{T}$ and $\Theta_{R}$ for the transmitter and the receiver, respectively ${ }^{1}$; (ii) the distance between $\mathrm{Tx}$ and $\mathrm{Rx}$ is much larger than the radii $R_{T}$ and $R_{R}$, i.e. $\max \left(R_{T}, R_{R}\right) \ll D$; (iii) for short periods of time, the mobile environment can be assumed as quasi-stationary, i.e. the rings of scatterers are fixed. The distances which a plane wave travels in the case of so-called double bounce scattering (Fig. 6 top) or single bounce scattering (Fig. 6 bottom) can be written as

$$
\begin{gathered}
D_{D B}=\left(D_{0}-R_{T} \cos \gamma_{T}^{m}+R_{R} \cos \gamma_{R}^{n}\right)+R_{R}+R_{T} \\
D_{S B T}=D_{0}-R_{T} \cos \gamma_{T}^{m}+R_{T}=D_{0}+2 R_{T} \sin ^{2} \frac{\gamma_{T}^{m}}{2}
\end{gathered}
$$

${ }^{1}$ Note that each scatterer $S_{T}^{m}\left(S_{R}^{n}\right)$ can be considered as a virtual basestation. Next, the communication link from each base station to the node can be modeled as a conventional fixed-to-mobile link. Hence, the properties of the channel between the nodes are strongly dependent on the scattering environment around each node. The concept of relative motion between Tx and Rx cannot be applied. 


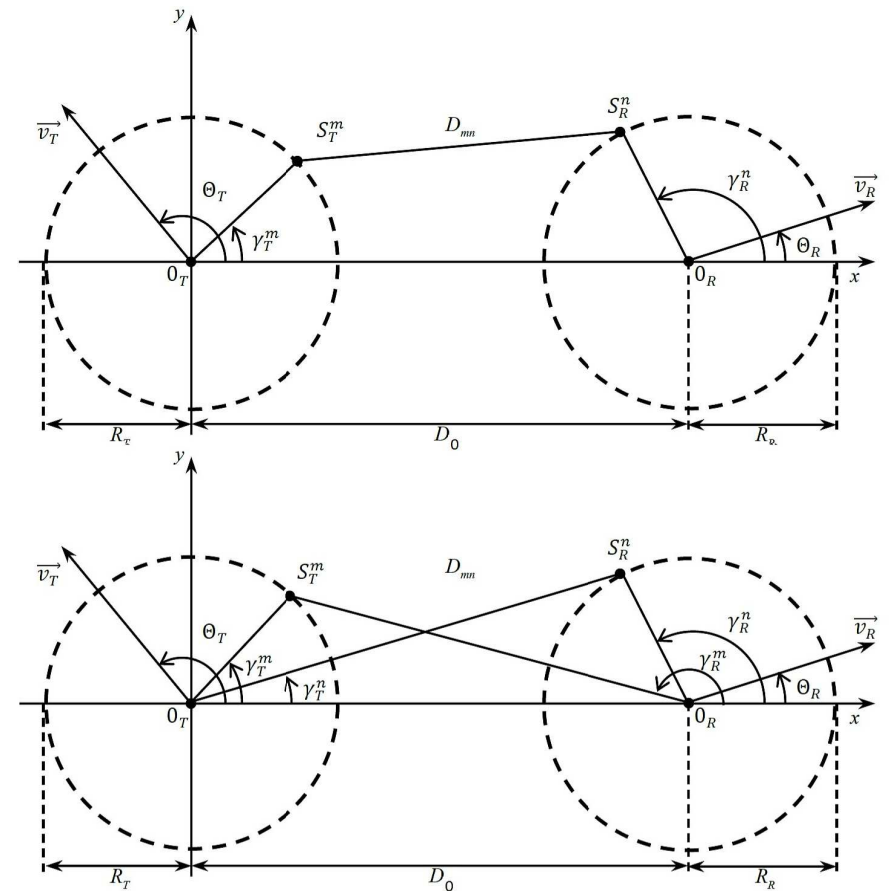

Fig. 6. The geometrical double-ring model with local scatterers around a mobile transmitter(left) and a mobile receiver(right) in case of the double bouncing(top) and the single bouncing(bottom)

$$
D_{S B R}=D_{0}+R_{R} \cos \gamma_{R}^{n}+R_{R}=D_{0}+2 R_{R} \cos ^{2} \frac{\gamma_{R}^{n}}{2},
$$

for scattering at the rings around the transmitter and receiver, respectively.

Based on this representation and extending a single-input and single-output (SISO) version of the reference model presented in [21] to the case of time-frequency fading, we can identify 3 types of mechanisms.

1) Line-of-Sight: The LOS component can be modeled as

$$
G_{L O S}(t, f)=e^{j\left(\frac{2 \pi f}{c}\left(t v_{T} \cos \Theta_{T}-t v_{R} \cos \Theta_{R}-D_{0}\right)+\psi_{L O S}\right)},
$$

where $\psi_{L O S}$ is a random phase shift over [0, $\left.2 \pi\right]$.

2) Double Bounce scattering: The component of the channel describing the transmission link under double bouncing conditions can be written as

$$
\begin{aligned}
& G_{D B}(t, f)= \\
& \lim _{\substack{N \rightarrow \infty \\
M \rightarrow \infty}} \frac{1}{\sqrt{M N}} \sum_{m=1}^{M} \sum_{n=1}^{N} e^{j\left(\frac{2 \pi f t}{c}\left(V_{T}+V_{R}\right)+\psi_{m n}+\phi_{n m}\right)},
\end{aligned}
$$

where $\psi_{m n}$ denotes the random phase-shift caused by the joint interaction of the scatterers $S_{T}^{m}$ and $S_{R}^{n}$ and $V_{T}$ and $V_{R}$ are described by

$$
\begin{gathered}
V_{T}=v_{T} \cos \left(\gamma_{T}^{m}-\Theta_{T}\right) \\
V_{R}=v_{R} \cos \left(\gamma_{R}^{n}-\Theta_{R}\right) .
\end{gathered}
$$

The phase shift $\phi_{n m}$ induced by the traveled distance does not depend on time $t$, and can be expressed as

$$
\phi_{n m}(f)=-\frac{2 \pi f}{c} D_{D B} .
$$

In [21], the double bounce component has only one phase per path and similar to (21). However, when the phase shifts introduced by each scatterer $S_{T}^{m}$ and $S_{R}^{n}$ are independent, i.e. $\psi_{m n}=\psi_{m}+\psi_{n}$, we can express (21) as a product of two single sums

$$
\begin{aligned}
& G_{D B I}(t, f)= \\
& \lim _{\substack{N \rightarrow \infty \\
M \rightarrow \infty}} \frac{1}{\sqrt{M N}} \sum_{m=1}^{M} e^{j\left(\frac{2 \pi f}{c}\left(V_{T} t-2 R_{T} \sin ^{2} \frac{\gamma_{T}^{m}}{2}\right)+\psi_{m}\right)} \\
& \quad \times \sum_{n=1}^{N} e^{j\left(\frac{2 \pi f}{c}\left(V_{R} t-2 R_{R} \cos ^{2} \frac{\gamma_{R}^{n}}{2}\right)+\psi_{n}\right)} .
\end{aligned}
$$

3) Single Bounce scattering: Using the approximations $\sin x \approx x\left(\right.$ for small $x$ ) and $\sin \left(\gamma_{R}^{m(n)}-\gamma_{T}^{m(n)}\right) \approx \sin \gamma_{T(R)}^{m(n)}$, we obtain the components caused by single bounce scattering as

$$
\begin{aligned}
& G_{S B T}(t, f)=\lim _{M \rightarrow \infty} \frac{1}{\sqrt{M}} \sum_{m=1}^{M} e^{j\left(\frac{2 \pi f t}{c}\left(V_{T}+\hat{V}_{R}\right)+\psi_{m}+\phi_{m}\right)} \\
& G_{S B R}(t, f)=\lim _{N \rightarrow \infty} \frac{1}{\sqrt{N}} \sum_{n=1}^{N} e^{j\left(\frac{2 \pi f t}{c}\left(\hat{V}_{T}+V_{R}\right)+\psi_{n}+\phi_{n}\right)}
\end{aligned}
$$

where $\hat{V}_{T}$ and $\hat{V}_{R}$ are given by

$$
\begin{gathered}
\hat{V}_{T}=\frac{v_{T} R_{R}^{2}}{2 D_{0}^{2}} \cos \left(2\left(\gamma_{R}^{n}-\Theta_{T}\right)\right) \\
\hat{V}_{R}=\frac{v_{R} R_{T}^{2}}{2 D_{0}^{2}} \cos \left(2\left(\gamma_{T}^{m}-\Theta_{R}\right)\right)
\end{gathered}
$$

The phase shift introduced by traveled distance

$$
\begin{aligned}
& \phi_{m}(f)=-\frac{2 \pi f}{c} D_{S B T} \\
& \phi_{n}(f)=-\frac{2 \pi f}{c} D_{S B R} .
\end{aligned}
$$

\section{B. Weights Identification}

The generalized reference model for SOSF can be obtained as a weighted combination of components described by (20), (21), (25), (26) and (27):

$$
\begin{aligned}
G(t, f) & =\kappa_{L O S} \cdot G_{L O S}(t, f) \\
& +\kappa_{S B R} \cdot G_{S B R}(t, f)+\kappa_{S B T} \cdot G_{S B T}(t, f) \\
& +\kappa_{D B} \cdot G_{D B}(t, f)+\kappa_{D B I} \cdot G_{D B I}(t, f)
\end{aligned}
$$

where the various $\kappa$ variables are the corresponding weights.

Since the central limit theorem states that each single sum is a zero-mean complex Gaussian process with unit variance, the envelopes $g_{D B}(t, f)=\left|G_{D B}(t, f)\right|, g_{S B R}(t, f)=$ $\left|G_{S B R}(t, f)\right|$ and $g_{S B T}(t, f)=\left|G_{S B T}(t, f)\right|$ are all characterized by Rayleigh distributions whereas the envelope $g_{D B I}(t, f)=\left|G_{D B I}(t, f)\right|$ enables to reproduce the DoubleRayleigh distribution, so that $\kappa_{D B I}$ can be identified to $\omega_{2}$ in (7). The Rayleigh term in (7) is made of the combination of all other components. In the absence of further information, we may set $\kappa_{D B}=\kappa_{S B T}=\kappa_{S B R}=\frac{\omega_{1}}{\sqrt{3}}$, assuming that all 
three mechanisms are occurring with the same probability ${ }^{2}$. Finally, $\kappa_{L O S}=\omega_{0}$, so that we can express (32) as

$$
\begin{aligned}
& G(t, f)=\omega_{0} \cdot G_{L O S}(t, f) \\
& +\frac{\omega_{1}}{\sqrt{3}} \cdot\left(G_{D B}(t, f)+G_{S B T}(t, f)+G_{S B R}(t, f)\right) \\
& +\omega_{2} \cdot G_{D B I}(t, f)
\end{aligned}
$$

where $\omega_{0}, \omega_{1}$ and $\omega_{2}$ can be experimentally derived or modeled using the concept described in Section III. From the statistical properties of (33), it can be concluded that smallscale time-frequency fading follows a SOSF distribution.

\section{Application to Double-and Single-Mobile Scenarios}

Let us now apply the generalized reference model to both types of mobility and consider only the temporal fading properties.

1) Temporal Fading in Double-Mobile Scenarios: Since $\psi_{n m}, \psi_{n}$ and $\psi_{m}$ are random, the constant (over time) shifts $\phi_{n m}, \phi_{m}$ and $\phi_{n}$ can be arbitrarily set to 0 . Furthermore, since $R_{T(R)} \ll D_{0}$, the impact of (28) and (29) is negligible when the node is mobile, the reference model under double-mobile conditions describes the fading behavior over time by

$$
\begin{gathered}
G_{D B}(t)=\lim _{\substack{N \rightarrow \infty \\
M \rightarrow \infty}} \frac{1}{\sqrt{M N}} \sum_{m=1}^{M} \sum_{n=1}^{N} e^{j\left(\frac{2 \pi f}{c}\left(V_{T} t+V_{R} t\right)+\psi_{m n}\right)} \\
G_{D B I}(t)=\lim _{\substack{N \rightarrow \infty \\
M \rightarrow \infty}} \frac{1}{\sqrt{M N}} \sum_{m=1}^{M} e^{j\left(\frac{2 \pi f}{c} V_{T} t+\psi_{m}\right)} \\
\times \sum_{n=1}^{N} e^{j\left(\frac{2 \pi f}{c} V_{R} t+\psi_{n}\right)} \\
G_{S B T}(t)=\lim _{M \rightarrow \infty} \frac{1}{\sqrt{M}} \sum_{m=1}^{M} e^{j\left(\frac{2 \pi f}{c} V_{T} t+\psi_{m}\right)} \\
G_{S B R}(t)=\lim _{N \rightarrow \infty} \frac{1}{\sqrt{N}} \sum_{n=1}^{N} e^{j\left(\frac{2 \pi f}{c} V_{R} t+\psi_{n}\right) .}
\end{gathered}
$$

Similar to the case of time-frequency fading, $g_{D B}(t)=$ $\left|G_{D B}(t)\right|, g_{S B R}(t)=\left|G_{S B R}(t)\right|$ and $g_{S B T}(t)=\left|G_{S B T}(t)\right|$ follow a Rayleigh distribution and the term $g_{D B I}(t)=$ $\left|G_{D B I}(t)\right|$ follows the Double-Rayleigh distribution, so that we can express (33) as

$$
\begin{aligned}
G_{d m}(t) & =\omega_{0} \cdot G_{L O S}(t) \\
& +\frac{\omega_{1}}{\sqrt{3}} \cdot\left(G_{D B}(t)+G_{S B T}(t)+G_{S B R}(t)\right) \\
& +\omega_{2} \cdot G_{D B I}(t)
\end{aligned}
$$

From the statistical properties of (38), it can be concluded that small-scale fading under double mobile conditions follows a SOSF distribution.

\footnotetext{
${ }^{2}$ It can be showed that the difference between the channels simulated using different impacts of $\kappa_{D B}, \kappa_{S B T}$ and $\kappa_{S B R}$ is negligible
}

2) Temporal Fading in Single-Mobile Scenarios: By setting the phase shifts $\phi_{n m}, \phi_{n}$ and $\phi_{m}$ to zero, the reference model under single-mobile conditions ${ }^{3}$ can be described by

$$
\begin{gathered}
G_{D B}(t)=\lim _{\substack{N \rightarrow \infty \\
M \rightarrow \infty}} \frac{1}{\sqrt{M N}} \sum_{m=1}^{M} \sum_{n=1}^{N} e^{j\left(\frac{2 \pi f}{c} V_{R} t+\psi_{m n}\right)} \\
G_{D B I}^{\prime}(t)=\lim _{\substack{N \rightarrow \infty \\
M \rightarrow \infty}} \frac{1}{\sqrt{M N}} \sum_{n=1}^{N} e^{j\left(\frac{2 \pi f}{c} V_{R} t+\psi_{n}\right)} \sum_{m=1}^{M} e^{j \psi_{m}}
\end{gathered}
$$

The component described by (37) remains unchanged in single mobile scenarios. Alternatively, $\hat{V}_{R}$ cannot be neglected in (26) since $V_{T}=0$. Consequently, (26) can be re-writed as

$$
G_{S B T}^{\prime}(t)=\lim _{N \rightarrow \infty} \frac{1}{\sqrt{N}} \sum_{n=1}^{N} e^{j\left(\frac{2 \pi f}{c} \hat{V}_{R} t+\psi_{n}\right)}
$$

So that we obtain

$$
\begin{aligned}
G_{s m}(t) & =\omega_{0} \cdot G_{L O S}(t) \\
& +\frac{\omega_{1}}{\sqrt{3}} \cdot\left(G_{D B}(t)+G_{S B T}^{\prime}(t)+G_{S B R}(t)\right) \\
& +\omega_{2} \cdot G_{D B I}^{\prime}(t)
\end{aligned}
$$

Again, the envelopes $g_{D B}(t)=\left|G_{D B}(t)\right|$ and $g_{S B R}(t)=$ $\left|G_{S B R}(t)\right|$ follow Rayleigh distributions since the central limit theorem states. However, since the second sum in (40) is constant over time, the envelope $g_{D B I}^{\prime}(t)=\left|G_{D B I}^{\prime}(t)\right|$ also follows a Rayleigh distribution, unlike double-mobile scenarios. The weight $\omega_{2}$ remains unchanged because it describes the impact of double bounce scattering with independent phase shifts introduced by each scatterer $S_{T}^{m}$ and $S_{R}^{n}$.

This means that in case of single mobility, the envelope of $g_{s m}(t)=\left|G_{s m}(t)\right|$ has a Rician (or Rayleigh) distribution.

\section{Reference Model Over Frequency}

Rather than investigating the temporal behavior at any given frequency, it is also possible to study the fading resulting from the reference model over frequency at any given time. This comes to express the frequency dependence of $\phi_{n m}, \phi_{m}, \phi_{n}$, so that we can rewrite the different components of the model as

$$
\begin{aligned}
& G_{D B}(f)= \\
& \lim _{\substack{N \rightarrow \infty \\
M \rightarrow \infty}} \frac{1}{\sqrt{M N}} \sum_{m=1}^{M} \sum_{n=1}^{N} e^{j\left(-\frac{4 \pi f}{c}\left(R_{T} \sin ^{2} \frac{\gamma_{T}^{m}}{2}+R_{R} \cos ^{2} \frac{\gamma_{R}^{n}}{2}\right)+\psi_{m n}\right)} \\
& G_{S B T}(f)=\lim _{M \rightarrow \infty} \frac{1}{\sqrt{M}} \sum_{m=1}^{M} e^{j\left(-\frac{4 \pi f}{c} R_{T} \sin ^{2} \frac{\gamma_{T}^{m}}{2}+\psi_{m}^{\prime}\right)} \\
& G_{S B R}(f)=\lim _{N \rightarrow \infty} \frac{1}{\sqrt{N}} \sum_{n=1}^{N} e^{j\left(-\frac{4 \pi f}{c} R_{R} \cos ^{2} \frac{\gamma_{R}^{n}}{2}+\psi_{n}^{\prime}\right)}
\end{aligned}
$$

${ }^{3}$ Only expressions for a fixed transmitter are shown in this subsection, but all expressions can similarly be applied to a fixed receiver by changing subscripts. 


$$
\begin{gathered}
G_{D B I}(f)=\lim _{\substack{N \rightarrow \infty \\
M \rightarrow \infty}} \frac{1}{\sqrt{M N}} \sum_{m=1}^{M} e^{j\left(-\frac{4 \pi f R_{T}}{c} \sin ^{2} \frac{\gamma_{T}^{m}}{2}+\psi_{m}\right)} \\
\times \sum_{n=1}^{N} e^{j\left(-\frac{4 \pi f R_{R}}{c} \cos ^{2} \frac{\gamma_{R}^{n}}{2}+\psi_{n}\right)}
\end{gathered}
$$

Since the central limit theorem states, the envelopes of $g_{D B}(f), g_{S B T}(f), g_{S B R}(f)$ are all characterized by Rayleigh distributions whereas the envelope of $g_{D B I}(f)$ follows a double-Rayleigh distribution.

Interestingly, the statistics of $(43-46)$ do not depend on the mobility. In other words, (46) can produce double-Rayleigh fading over frequency even for a single-mobile scenario. Probably, this fact could be the reason for apparent double-Rayleigh fading measurements in single-mobile scenarios in [20], [22], which are based on the same measurement campaign. Indeed, in these models, the fading behavior is characterized by jointly considering time samples and frequency tones, although we have shown above that they might not follow the same distribution in single-mobile scenarios.

\section{E. Second-Order Statistics of SOSF channels}

We have outlined in Section III-A that a double-ring geometry could be used to reproduce the SOSF first-order statistics observed in measurements. Therefore, we can use the same geometrical model to infer the second-order statistics (the autocorrelation function), which could not be extracted from our measurements.

1) Second-Order Statistics in Double Mobile Scenarios: In double-mobile scenarios, the correlation properties of the reference channel model can be summarized as

$$
\begin{aligned}
R_{G_{i} G_{i}}(\tau) & =R_{G_{q} G_{q}}(\tau)=\mathbb{E}\left[G_{q}(t) G_{q}(t+\tau)\right] \\
& =\omega_{0}^{2} \cos \left(2 \pi \tau\left(f_{T} \cos \Theta_{T}-f_{R} \cos \Theta_{R}\right)\right) \\
& +\frac{\omega_{1}^{2}}{3}\left(J_{0}\left(2 \pi f_{T} \tau\right)+J_{0}\left(2 \pi f_{R} \tau\right)\right) \\
& +\left(\frac{\omega_{1}^{2}}{3}+\omega_{2}^{2}\right) J_{0}\left(2 \pi f_{T} \tau\right) J_{0}\left(2 \pi f_{R} \tau\right) \\
R_{G_{i} G_{q}}(\tau) & =-R_{G_{q} G_{i}}(\tau)= \\
& =\omega_{0}^{2} \sin \left(2 \pi \tau\left(f_{T} \cos \Theta_{T}-f_{R} \cos \Theta_{R}\right)\right) \\
R_{G G}(\tau) & =\mathbb{E}\left[G(t) G^{*}(t+\tau)\right]= \\
& =\omega_{0}^{2} e^{\left(j 2 \pi \tau\left(f_{T} \cos \Theta_{T}-f_{R} \cos \Theta_{R}\right)\right)} \\
& +\frac{\omega_{1}^{2}}{3}\left(J_{0}\left(2 \pi f_{T} \tau\right)+J_{0}\left(2 \pi f_{R} \tau\right)\right) \\
& +\left(\frac{\omega_{1}^{2}}{3}+\omega_{2}^{2}\right) J_{0}\left(2 \pi f_{T} \tau\right) J_{0}\left(2 \pi f_{R} \tau\right)
\end{aligned}
$$

where $G=G_{i}+j G_{q}$, and $f_{R}, f_{T}$ and $f_{r e l}$ denote the Doppler frequencies caused by motion of the nodes,

$$
\begin{aligned}
& f_{R}=\frac{v_{R} \cdot f}{c} \\
& f_{T}=\frac{v_{T} \cdot f}{c} .
\end{aligned}
$$

By proceeding as in [21], we can obtain the proofs for the derivations of the auto- and cross-correlations.
2) Second-Order Statistics in Single Mobile Scenarios: By using [21], it can be showed that the correlation functions of the components described by (37), (39) and (40) are the same. Using $[18, \S 3.715 .13, \S 3.715 .18]$ to derive the correlations of the component (41), we can write:

$$
\begin{aligned}
& R_{G_{i} G_{i}}(\tau)=R_{G_{q} G_{q}}(\tau)=\mathbb{E}\left[G_{q}(t) G_{q}(t+\tau)\right] \\
& =\omega_{0}^{2} \cos \left(2 \pi \tau\left(f_{T} \cos \Theta_{T}-f_{R} \cos \Theta_{R}\right)\right) \\
& +\left(\frac{2 \omega_{1}^{2}}{3}+\omega_{2}\right) \cdot J_{0}\left(2 \pi f_{m} \tau\right)+\frac{\omega_{1}^{2}}{3} \cdot J_{0}\left(\frac{R^{2}}{D^{2}} \pi f_{m} \tau\right) \\
& R_{G_{i} G_{q}}(\tau)=-R_{G_{q} G_{i}}(\tau)= \\
& =\omega_{0}^{2} \sin \left(2 \pi \tau\left(f_{T} \cos \Theta_{T}-f_{R} \cos \Theta_{R}\right)\right) \\
& R_{G G}(\tau)=\mathbb{E}\left[G(t) G^{*}(t+\tau)\right]= \\
& =\omega_{0}^{2} e^{\left(j 2 \pi \tau\left(f_{T} \cos \Theta_{T}-f_{R} \cos \Theta_{R}\right)\right)} \\
& +\left(\frac{2 \omega_{1}^{2}}{3}+\omega_{2}\right) \cdot J_{0}\left(2 \pi f_{m} \tau\right) \\
& +\frac{\omega_{1}^{2}}{3} \cdot J_{0}\left(\frac{R^{2}}{D^{2}} \pi f_{m} \tau\right)
\end{aligned}
$$

where $f_{m}$ denote the Doppler frequency caused by motion of the mobile node

$$
f_{m}= \begin{cases}f_{T} & {\left[v_{R}=0\right]} \\ f_{R} & {\left[v_{T}=0\right]}\end{cases}
$$

\section{TIME-SERIES MODEL}

\section{A. Path Loss and Mean Shadowing}

Considering the expression for the received power (3) and shadowing (4), the combined path-loss and mean shadowing $L=\Lambda+\bar{S}$ can be defined as

$$
L=\Lambda_{0}+\eta \cdot 10 \log _{10}\left(\frac{d}{d_{0}}\right)+\bar{S}
$$

where $\Lambda_{0}$ is the deterministic path-loss at the reference distance $d_{0}=1 \mathrm{~m}$ and $\bar{S}$ is found to be normal with zero mean (when expressed in decibels) over the whole set of rooms/links. The deterministic path loss at the reference distance $\Lambda_{0}$, the path loss exponent $\eta$ and the standard deviation $\sigma_{\bar{s}}$ characterizing distribution of $\bar{S}$ have been estimated from the data (see Table VI).

\section{B. Dynamic Shadowing}

When the deterministic path-loss and mean shadowing are removed and small-scale fading is averaged out, the remaining variations are due to dynamic shadowing, as detailed earlier. Again, we observe in Fig. 7 that the dynamic shadowing might be described by a t-location scale distribution

$p\left(x \mid \nu, \mu, \sigma_{\tilde{s}}\right)=\frac{\Gamma\left(\frac{\nu+1}{2}\right)}{\Gamma\left(\frac{\nu}{2}\right) \sigma_{\tilde{s}} \sqrt{\pi \nu}}\left(1+\frac{1}{\nu}\left(\frac{x-\mu}{\sigma_{\tilde{s}}}\right)^{2}\right)^{-\frac{\nu+1}{2}}$

with zero-mean $(\mu=0)$ by definition, $\nu=5$ degrees of freedom and a scale parameter $\sigma_{\tilde{s}}$ (see Table VI). This distribution results from compounding a normal distribution with mean $\mu$ and unknown variance, with an inverse gamma distribution placed over the variance with parameters $\alpha_{\Gamma}=\nu / 2$ and 


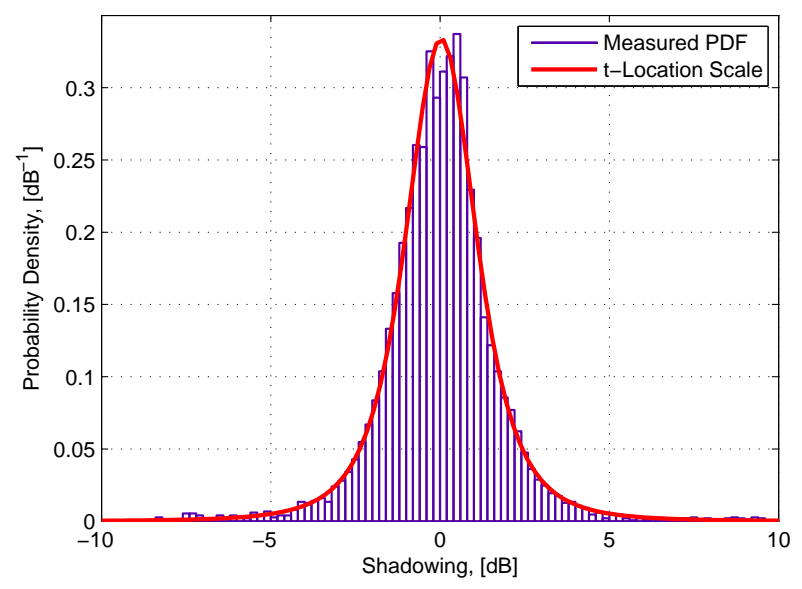

Fig. 7. Fit of the T-location scale distribution to measured data in a singlemobile, small-scale motion scenario

TABLE VI

MODEL PARAMETERS FOR PATH-LOSS AND SHADOWING

\begin{tabular}{l|l|l|l|l|l|l}
\hline Scenario & $\sigma_{\tilde{s}}$ & $\tau_{\tilde{s}}[\mathrm{~s}]$ & $\nu$ & $\Lambda_{0}[\mathrm{~dB}]$ & $\eta$ & $\sigma_{\bar{s}}$ \\
\hline Single-mobile, small-scale motion & 1.1 & & & & & \\
Single-mobile, large-scale motion & 1.6 & \multirow{2}{*}{6.3} & 5 & 42 & 2.8 & 9.8 \\
Double-mobile, small-scale motion & 1.8 & & & & & \\
Double-mobile, large-scale motion & 2.5 & & & & & \\
\hline
\end{tabular}

$\beta_{\Gamma}=\nu \sigma_{\tilde{s}}^{2} / 2$. In other words, the dynamic shadowing is assumed to have a Gaussian distribution with an unknown variance distributed as inverse gamma, and then the variance is marginalized out. As it was mentioned in Section II-C, different mechanizmes typical for investigated scenarios lead to variations of the value of the scale parameter $\sigma_{\tilde{s}}$ depending on the type of mobility and the motion scale.

The dynamic shadowing autocorrelation over time is modeled as a decreasing exponential, whose decay time $\tau_{\tilde{s}}$ is also shown in Table VI. Hence, we use an autoregressive process to generate autocorrelated dynamic shadowing values,

$$
\tilde{S}(t)=e^{-1 / \tau_{\tilde{s}}} \tilde{S}(t-1)+\sqrt{1-e^{-2 / \tau_{\tilde{s}}}} x_{\tilde{s}}(t)
$$

where $x_{\tilde{s}}$ is a time series of length $T$, whose values are drawn independently from the distributions described by Table VI.

No correlation between dynamic shadowing and the smallscale fading states has been found, so that we can combine independently simulated dynamic shadowing and small-scale fading realizations.

\section{Small-Scale Fading}

Summarizing, we propose to model peer-to-peer channels with time-variant statistics as shown in Fig. 8. We start with a random initial state of the HMM. Next, using the corresponding distribution provided in Table III, we draw the SOSF parameters $(\alpha, \beta)$. Using a weighted combination (the weights are extracted using expressions $(17-19)$ ) of correlated fast fading realizations generated by the model ${ }^{4}$ described in [13],

\footnotetext{
${ }^{4}$ Filter coefficients used in the model are specified by appropriate first- and
} second-order statistics derived in Section IV-E.

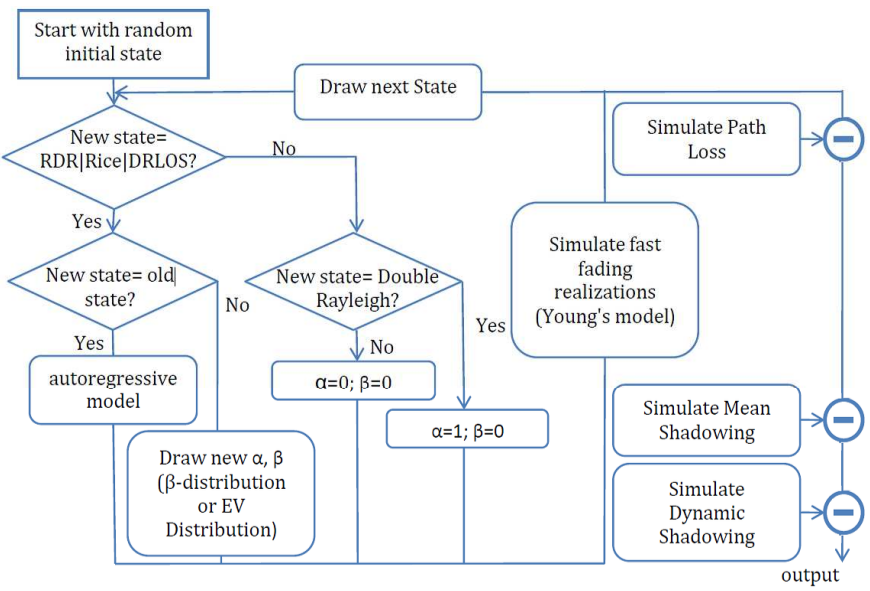

Fig. 8. Flow-diagram of the simulation model

we obtain a pre-defined number of correlated complex fading realizations $G(t)$. Next, the path-loss, the mean shadowing and the dynamic shadowing are added to small-scale fading realizations. Next, the state of the HMM is updated. If the state is unchanged, we use the autoregressive models described by $(14-16)$ to generate new values of $\alpha, \beta$ and $\Delta$ for RDR, Rician or DRLOS subsets or we keep the constant values of the parameters for Rayleigh and double-Rayleigh subsets. If the state is modified, depending on the subset, we generate the parameters $\alpha$ and $\beta$ using the distributions described in Table III or we set fixed values (for Rayleigh and double-Rayleigh subsets). Note that duration of one state equals $\frac{113[\mathrm{~s}]}{T}=0.8 \mathrm{~s}$.

\section{Simulations and Validation}

Modeled time-variant fading statistics of a peer-to-peer network in an indoor office environment are shown in Fig. 9 (top). The first five pairs $(\alpha, \beta)$ are then used to simulate channel
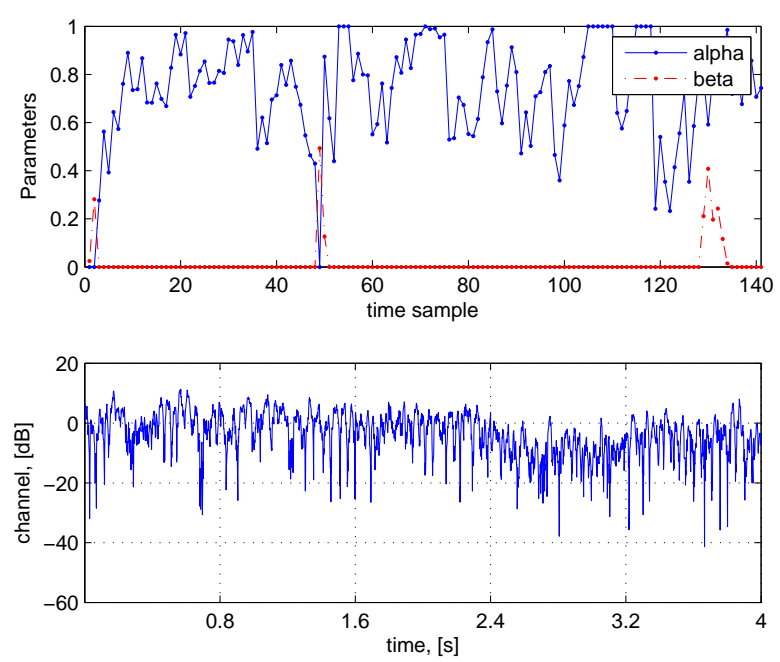

Fig. 9. Simulated small-scale fading distribution parameters over time for the double-mobile scenario (top). Simulated time-variant components of the channel (fast fading and dynamic shadowing) (bottom) 


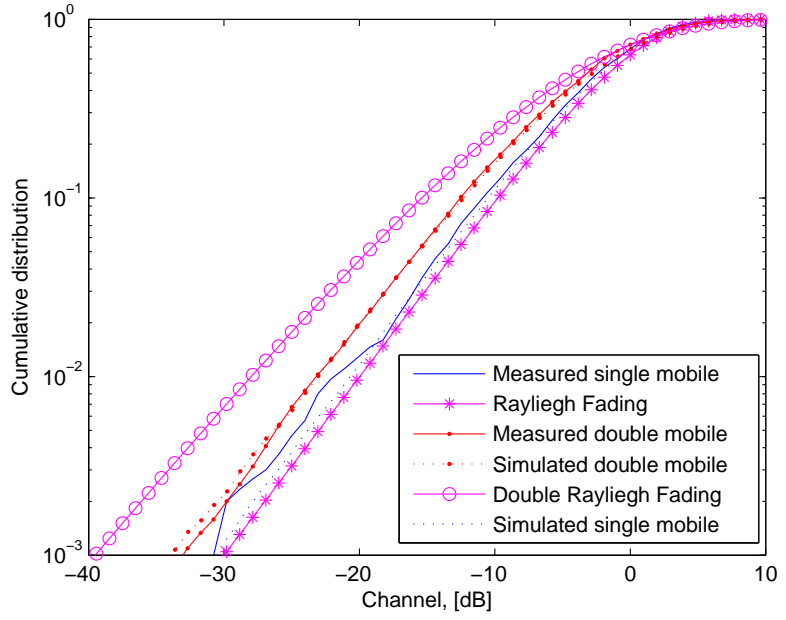

Fig. 10. Fit of the model to measured data

realizations (only the combination of small-scale fading and dynamic shadowing are illustrated) in Fig. 9 (bottom).

Fig. 10 compares the statistics of two sets of measured channels and generated channels using the model of Section $\mathrm{V}-\mathrm{C}$. To verify the model under different mobile conditions, we simulated sets of single-mobile links between nodes separated by the distance $d=12 \mathrm{~m}$ and between mobile nodes separated by the distance $d=13 \mathrm{~m}$. Next, we compared the cumulative distribution function (CDF) of the generated fast fading with the $\mathrm{CDF}$ of measured fast fading between nodes having the same parameters (Rx1-Tx7,Rx7-Tx1 and Rx7-Tx8 for singlemobile and double-mobile scenarios respectively). To quantify the difference between CDFs of measured and simulated fast fading, the mean squared error (MSE) was estimated for both mobility scenarios. The asymptotic values of MSE $\left(7.8 \cdot 10^{-5}\right.$ for single mobile and $6 \cdot 10^{-5}$ for double mobile curves) are achieved after 50 simulation trials. The bigger value of MSE for the simulation under single mobile conditions can be explained by the fact that we cannot use realizations of fast fading measured on different frequency tones(see Section IV-C) to obtain CDF of fast fading under single mobile conditions. Probably, this explains the nonsmooth behavior of the CDF below $-20 \mathrm{~dB}$ level.

Fig. 11 shows normalized level crossing rates (LCR) of the simulated time-variant components of the channel(fast fading and dynamic shadowing) for both types of mobility and different motion scales in comparison with theoretical curves. It can be noted that simulation results are different from the conventional methods. The comparison of the measured and simulated LCR cannot be provided since we cannot extract LCR from measurements.

\section{CONCLUSION}

This paper has presented an analysis and an empirical model of time-variant channel statistics of a peer-to-peer network based on measurement in an indoor office environment at 3.8 GHz:

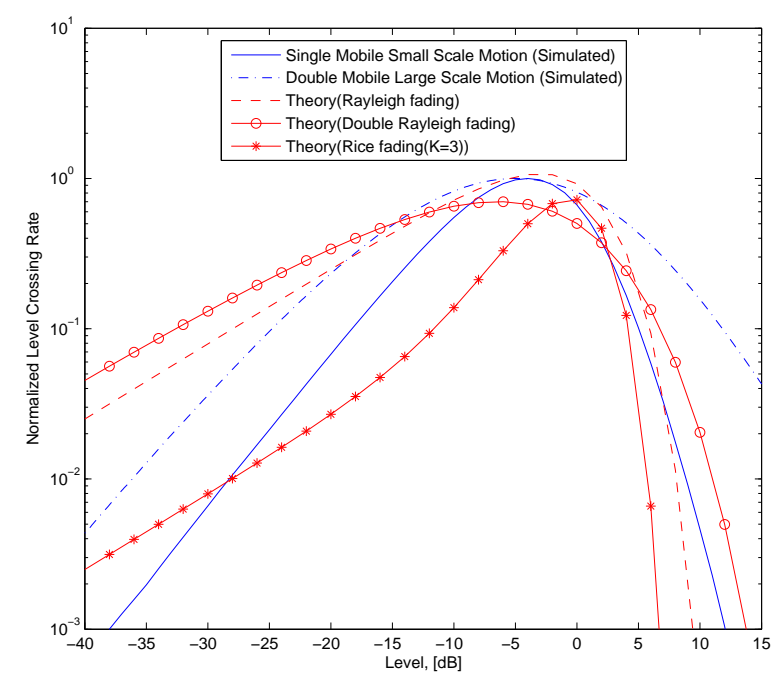

Fig. 11. Level crossing rate normalized to the maximum Doppler frequency

1) Models and simulators for second-order scattering fading (SOSF) in different mobility scenarios have been proposed.

2) In double-mobile scenarios, the measured data is characterized by the SOSF distribution, with time-variant statistics ranging from double-Rayleigh to Rician.

3) In single-mobile scenarios, temporal small-scale fading is Rayleigh or Rician distributed.

4) Frequency small-scale fading does not depend on mobility and follows a SOSF distribution.

5) Distributions of the parameter $\alpha$ for the Rayleigh-double Rayleigh and $\Delta$ for the double-Rayleigh-LOS subsets can be well approximated by the Beta distribution.

6) Distribution of the K-Factor can be modeled by Extremal Value distribution.

7) Transitions between the fading states are described by a hidden Markov model.

8) Following the concept of [4], mean shadowing and dynamic shadowing are separated. We find that the experimental dynamic shadowing distribution follows the t-location scale distribution.

9) The proposed model has been validated and good agreement between simulations using the proposed model and measurement data is obtained

\section{REFERENCES}

[1] R. Nabar, H. Bolcskei, and F. Kneubuhler, "Fading relay channels: performance limits and space-time signal design," IEEE Journal on Selected Areas in Communications, vol. 22, no. 6, pp. 1099-1109, Aug 2004.

[2] J. Laneman and G. W. Wornell, "Distributed space-time-coded protocols for exploiting cooperative diversity in wireless networks," IEEE Transactions on Information Theory, vol. 49, no. 10, pp. 2415-2425, Oct 2003.

[3] H. Ochiai, P. Mitran, H. Poor, and V. Tarokh, "Collaborative beamforming for distributed wireless ad hoc sensor networks," IEEE Transactions on Signal Processing, vol. 53, no. 11, pp. 4110-4124, Nov 2005. 
[4] C. Oestges, N. Czink, B. Bandemer, P. Castiglione, F. Kaltenberger, and A. Paulraj, "Experimental characterization and modeling of outdoor-toindoor and indoor-to-indoor distributed channels," IEEE Transactions on Vehicular Technology, vol. 59, no. 5, pp. 2253-2265, Jun 2010.

[5] M. Le Treust and S. Lasaulce, "A repeated game formulation of energyefficient decentralized power control," IEEE Transactions on Wireless Communications, vol. 9, no. 9, pp. 2860-2869, September 2010.

[6] J. Karedal, A. Johansson, F. Tufvesson, and A. Molisch, "A measurement-based fading model for wireless personal area networks," IEEE Transactions on Wireless Communications, vol. 7, no. 11, pp. 4575-4585, November 2008.

[7] I. Cuinas and M. Sanchez, "Measuring, modeling, and characterizing of indoor radio channel at $5.8 \mathrm{GHz}$," Vehicular Technology, IEEE Transactions on, vol. 50, no. 2, pp. 526-535, Mar 2001.

[8] N. Czink, B. Bandemer, G. Vazquez-Vilar, L. Jalloul, C. Oestges, and A. Paulraj, "Spatial separation of multi-user MIMO channels," IEEE 20th International Symposium on Personal, Indoor and Mobile Radio Communications (PIMRC), pp. 1059-1063, Sept 2009.

[9] I. Kovacs, P. C. F. Eggers, K. Olesen, and L. Petersen, "Investigations of outdoor-to-indoor mobile-to-mobile radio communication channels," Vehicular Technology Conference, 2002. Proceedings. VTC 2002-Fall. 2002 IEEE 56th, vol. 1, pp. 430-434 vol.1, 2002.

[10] B. Clerckx and C. Oestges, MIMO Wireless Networks. 2nd ed. Elsevier Academic Press

[11] C. Patel, G. Stuber, and T. Pratt, "Simulation of Rayleigh-faded mobileto-mobile communication channels," IEEE Transactions on Communications, vol. 53, no. 11, pp. 1876-1884, Nov 2005.

[12] Y. Ephraim and N. Merhav, "Hidden Markov processes," IEEE Transac tions on Information Theory, vol. 48, no. 6, pp. 1518-1569, Jun 2002

[13] D. Young and N. Beaulieu, "The generation of correlated Rayleigh random variates by inverse discrete fourier transform," IEEE Transactions on Communications, vol. 48, no. 7, pp. 1114-1127, Jul 2000.

[14] C. Oestges, P. Castiglione, and N. Czink, "Empirical modeling of nomadic peer-to-peer networks in office environment," in Vehicular Technology Conference (VTC Spring), 2011 IEEE 73rd, May 2011, pp. $1-5$.

[15] J. D. Parsons, The Mobile Radio Propagation Channel. 2nd ed. London, U.K.:Wiley.

[16] "Propagation data and prediction models for the planning of indoor radiocommunication systems and radio local area networks in the frequency range $900 \mathrm{MHz}$ to $100 \mathrm{GHz}$,' Recommendation ITU-R P.1238 4, 2005

[17] J. Salo, H. El-Sallabi, and P. Vainikainen, "Statistical analysis of the multiple scattering radio channel," IEEE Transactions on Antennas and Propagation, vol. 54, no. 11, pp. 3114-3124, Nov 2006.

[18] I. Gradshtein and I. Ryzhik, Table of integrals, series, and products 7th ed. Academic Press, Inc.

[19] B. Bandemer, C. Oestges, N. Czink, and A. Paulraj, "Physically motivated fast-fading model for indoor peer-to-peer channels," Electronics Letters, vol. 45, no. 10, pp. 515-517, May 2009.

[20] M. Gan, N. Czink, P. Castiglione, C. Oestges, F. Tufvesson, and T. Zemen, "Modeling time-variant fast fading statistics of mobile peerto-peer radio channels," Vehicular Technology Conference (VTC Spring), 2011 IEEE 73rd, pp. 1-5, May 2011.

[21] A. Zajic and G. Stuber, "Space-time correlated mobile-to-mobile channels: Modelling and simulation," Vehicular Technology, IEEE Transactions on, vol. 57, no. 2, pp. 715-726, March 2008.

[22] F. Sanchez, A. Stephanides, N. Czink, and C. Oestges, "Fast fading characterization for indoor to indoor and outdoor to indoor channels," Vehicular Technology Conference (VTC Fall), 2011 IEEE, pp. 1-5, Sept 2011. 